\title{
Model-Assisted Fine-Tuning of Central Carbon Metabolism in Yeast through dCas9- Based Regulation
}

Ferreira, Raphael; Skrekas, Christos; Hedin, Alex; Sánchez, Benjamín José; Siewers, Verena; Nielsen, Jens; David, Florian

Published in:

ACS Synthetic Biology

Link to article, DOI:

10.1021/acssynbio.9b00258

Publication date:

2019

Document Version

Publisher's PDF, also known as Version of record

Link back to DTU Orbit

Citation (APA):

Ferreira, R., Skrekas, C., Hedin, A., Sánchez, B. J., Siewers, V., Nielsen, J., \& David, F. (2019). Model-Assisted Fine-Tuning of Central Carbon Metabolism in Yeast through dCas9-Based Regulation. ACS Synthetic Biology, 8 , 2457-2463. https://doi.org/10.1021/acssynbio.9b00258

\section{General rights}

Copyright and moral rights for the publications made accessible in the public portal are retained by the authors and/or other copyright owners and it is a condition of accessing publications that users recognise and abide by the legal requirements associated with these rights.

- Users may download and print one copy of any publication from the public portal for the purpose of private study or research.

- You may not further distribute the material or use it for any profit-making activity or commercial gain

- You may freely distribute the URL identifying the publication in the public portal 


\section{Model-Assisted Fine-Tuning of Central Carbon Metabolism in Yeast through dCas9-Based Regulation}

Raphael Ferreira, ${ }^{\dagger, \ddagger}$ Christos Skrekas, ${ }^{\dagger, \ddagger}$ Alex Hedin, $^{\dagger}$ Benjamín J. Sánchez, ${ }^{\dagger \neq}$ Verena Siewers, ${ }^{\dagger, \ddagger}$ Jens Nielsen, ${ }^{\dagger, \ddagger, \$}$ and Florian David ${ }^{*,+,}$

${ }^{\dagger}$ Department of Biology and Biological Engineering, Chalmers University of Technology, SE412 96 Gothenburg, Sweden

${ }^{\ddagger}$ Novo Nordisk Foundation Center for Biosustainability, Chalmers University of Technology, SE412 96 Gothenburg, Sweden

${ }^{\S}$ Novo Nordisk Foundation Center for Biosustainability, Technical University of Denmark, DK2800 Kgs. Lyngby, Denmark

Supporting Information

ABSTRACT: Engineering Saccharomyces cerevisiae for industrial-scale production of valuable chemicals involves extensive modulation of its metabolism. Here, we identified novel gene expression fine-tuning set-ups to enhance endogenous metabolic fluxes toward increasing levels of acetyl-CoA and malonyl-CoA. dCas9-based transcriptional regulation was combined together with a malonyl-CoA responsive intracellular biosensor to select for beneficial set-ups. The candidate genes for screening were predicted using a genome-scale metabolic model, and a gRNA library targeting a total of 168 selected genes was designed. After multiple rounds of fluorescence-activated cell sorting and library sequencing, the gRNAs that were functional and increased flux toward malonyl-CoA were assessed for their efficiency to enhance 3-hydroxypropionic acid (3-HP) production. 3-HP production was significantly improved upon fine-tuning genes involved in providing malonyl-CoA precursors, cofactor supply, as well as chromatin remodeling.

KEYWORDS: synthetic biology, biosensor, CRISPR, flux balance analysis
Sints ince Saccharomyces cerevisiae is a well-studied, industrially robust microorganism, it is feasible to engineer its metabolism for overproduction of chemicals reaching industrial standards. ${ }^{1,2}$ Genome engineering to introduce genetic modifications such as gene deletion, overexpression, and exact regulation is commonly utilized to improve pathway efficiency and product yield. In this context, inverse metabolic engineering is used to uncover new targets via high-throughput screening of random genetic libraries. Inverse metabolic engineering relies on the selection of a high-producing genotype from a diverse, previously generated population of cells. However, screening for increased production of a biomolecule can be challenging in high throughput, as large amounts of biomass from each genotype are needed for traditional analyses like mass spectrometry. One approach to improve throughput is the use of genetically encoded biosensors, coupled to a fluorescence output, that enable single cell, real-time monitoring of cellular metabolism and screening of large diversified libraries using fluorescenceactivated cell sorting (FACS). ${ }^{3}$ However, given that there are thousands of genes whose up- or downregulation could potentially increase or decrease the final product yield, and expression of each of those genes would need be tuned to an optimal level, it remains challenging to determine which genes

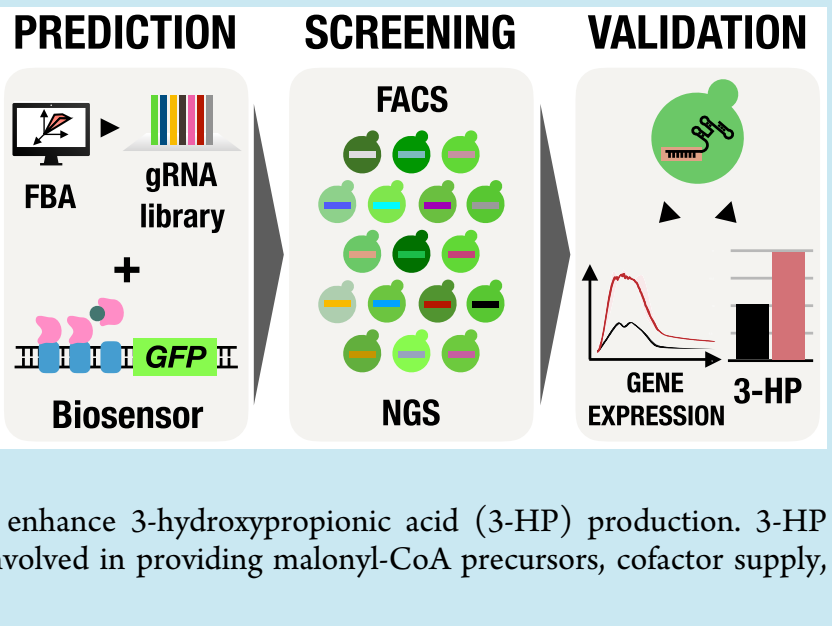

SCREENING

pubs.acs.org/synthbio 


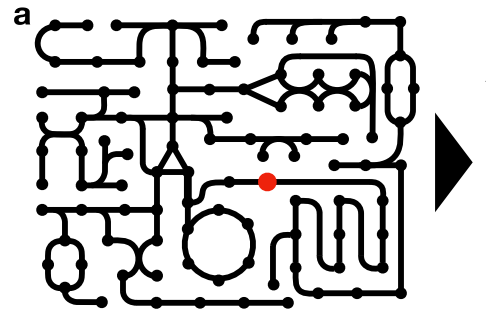

Genome-Scale Metabolic Model Yeast 7.6

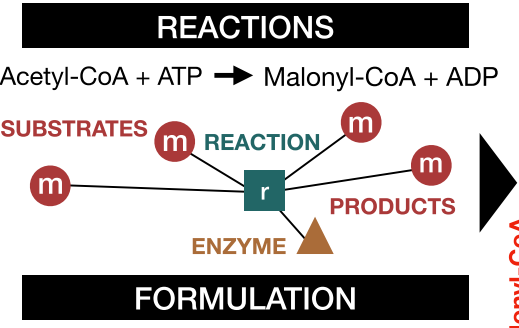

$\mathbf{S}=\left(\begin{array}{ccccc}r_{1} & r_{2} & r_{3} & & r_{n} \\ 1 & 1 & 0 & \cdots & 0 \\ -1 & 0 & 0 & & 0 \\ \vdots & \vdots & \vdots & & \vdots \\ 0 & 1 & 0 & \cdots & 0\end{array}\right) \mathrm{m}_{1}$

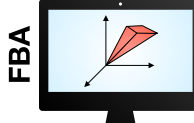

11 OBJECTIVES: GROWTH

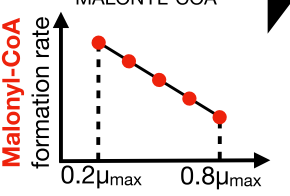

Biomass formation rate Constraints: Sv $=0 \quad \mathrm{lb}<\mathrm{v}<\mathrm{ub}$ $\mu=\alpha \cdot \mu_{\max }$

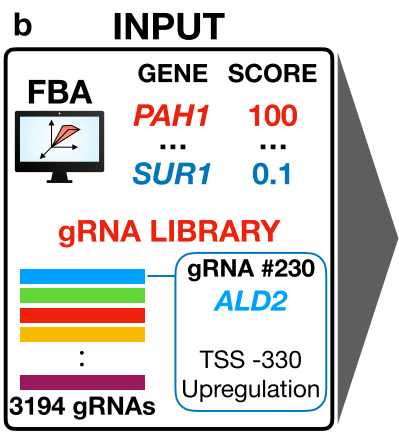

SELECTION

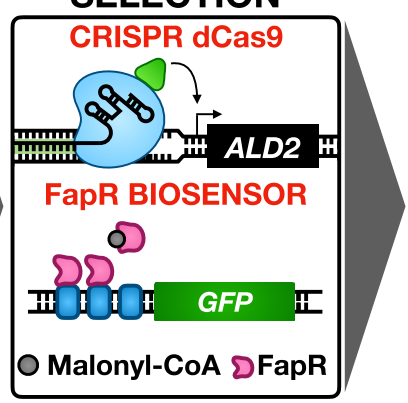

SCREENING

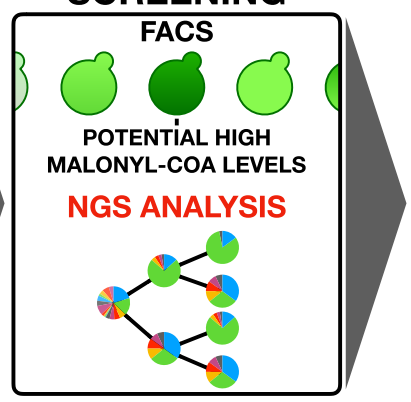

VALIDATION

3-HP PRODUCTION

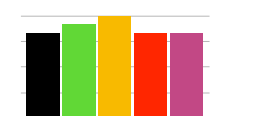

GENE REGULATION

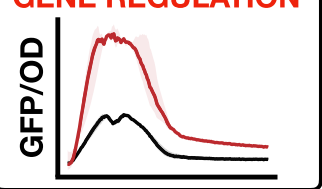

Figure 1. Overview of the framework for model-assisted fine-tuning through dCas9-based regulation. (a) Genome-scale metabolic model fundamentals where each metabolic reaction and its constituents is mathematically represented in a global model. Here, we apply FBA maximizing the production of malonyl-CoA as the objective function of the model. Fluxes toward malonyl-CoA are computationally evaluated at different growth rates, and a list of genes potentially contributing to enhancing malonyl-CoA production is retrieved. A target score for upregulation or downregulation is associated with each gene as a proxy for fine-tuning. Here, for all $\alpha$ 's and all reactions, the scoring is defined as the average of the flux $_{\alpha}$ over flux $\mathrm{WT}_{\mathrm{WT}}$. (b) Workflow of the study. On the basis of the gene scores obtained by the FBA, a gRNA library of 3194 gRNAs is designed to target 168 genes. The gRNA library is coupled to a CRISPR-dCas9-VPR and a FapR-based malonyl-CoA biosensor, which allows transcriptional perturbation of the selected genes and an output screening. Then, multiple rounds of fluorescence-based sorting and NGS is applied to retrieve the most efficient gRNA to enhance fluxes toward malonyl-CoA based on the fluorescence levels. Finally, the most enriched gRNAs are individually assessed on their efficiency to enhance 3-HP production as well as transcriptional regulation by coupling their targeted promoter to GFP.

library and a malonyl-CoA responsive intracellular biosensor in yeast to identify genes that potentially contribute to enhancing the flux toward cytosolic malonyl-CoA. ${ }^{10,11}$ The candidate genes for screening were predicted by constraint-based flux balance analysis (FBA) and altered transcription levels were established using dCas9 coupled to the VP64-p65-Rta (VPR) tripartite activator, which can enable upregulation or downregulation depending on the gRNA position. ${ }^{12,13}$ A gRNA library of 3194 gRNAs targeting a total of 168 selected genes was constructed and screened for potential malonyl-CoA overproducers through FACS. gRNAs enriched among highly fluorescent cells were subsequently assessed on their effect on transcriptional regulation by coupling their targeted promoter to GFP, as well as their efficiency to enhance production of 3hydroxypropionic acid (3-HP), a product derived from malonyl-CoA.

\section{RESULTS}

Flux Balance Analysis Allows for Prediction of Target Genes for dCas9-Based Regulation. We started with an in silico constraint-based modeling approach for predicting a set of genes in S. cerevisiae that have the highest impact on the production of malonyl-CoA, an endogenous precursor for several industrially relevant biochemicals. ${ }^{11} \mathrm{We}$ performed FBA for two carbon sources, glucose and ethanol, using as objective function a combination of the specific growth rate $\left(\mu_{\max }\right)$ and acetyl or malonyl production (Figure 1a, Table S1-
S2).${ }^{14}$ For each simulation and each reaction in the metabolic network, we computed a so-called $k$-score that compared the corresponding flux in the simulation with the flux of the same reaction at maximum growth rate conditions. This way, scores larger than 1 represent an increased flux for that specific reaction as acetyl/malonyl-CoA production increases, which points toward an upregulation candidate; on the other hand, $k$ scores lower than 1 represent a corresponding decrease in the flux, which corresponds to a downregulation candidate (Figure 1a).

The analysis yielded 168 genes as potential candidates for upregulation or downregulation in cytosolic acetyl or malonyl overproduction in growth in glucose or ethanol as carbon source. 70 of those genes were found to be targets for upregulation, 80 for downregulation, and 18 found to be either upregulation or downregulation targets depending on the carbon source (Table S2). A flux variability analysis (FVA) yielded that over $95 \%$ of these candidates show this upregulation/downregulation in a consistent manner. ${ }^{15}$ From Saccharomyces Genome Database (SGD) GO Term Finder analysis, most of the selected genes encode proteins that display a transmembrane transporter activity, and/or represent cofactor binding enzymes (Table S3). ${ }^{16}$

Malonyl-CoA Biosensor Assisted Sorting of a gRNA Library Leads to Enrichment in Specific gRNAs. Disruption of Cas9 endonuclease domains (RuvC ${ }^{\text {D10A }}$ along with $\mathrm{HNH}^{\mathrm{H} 840 \mathrm{~A}}$ ) results in a catalytically inactive enzyme that 

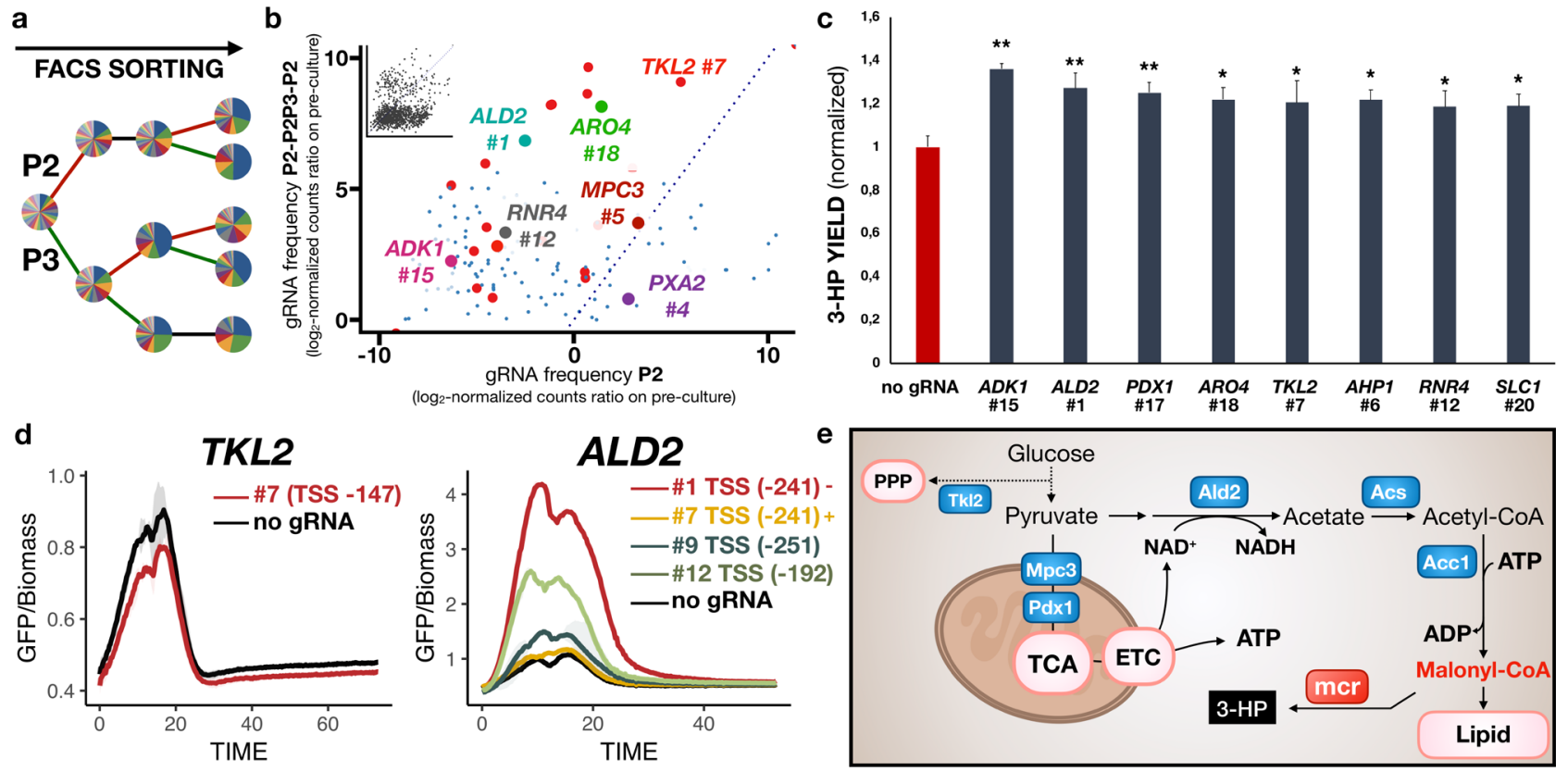

Figure 2. Expression fine-tuning with a dCas9-based gRNA library allows enhancing fluxes toward malonyl-CoA. (a) Multiple FACS sorting at different fluorescence gates (P2 and P3). gRNA enrichment throughout multiple rounds of FACS and NGS. Pie charts show enriched gRNAs between different rounds of sorting. Each color represents one gRNA. (b) Scatter plot for visualizing gRNA enrichment. Shown on the $y$-axis: base 2 log fold-change gRNAs of sorting P2-P2P3-P2 over the initial library (preculture), and shown on the $x$-axis: base 2 log fold-change gRNAs of sorting P2 over the initial library (preculture). Shown in large colored circles are the enriched gRNAs that were tested for 3-HP production and in small blue dots the rest of the library. (c) Some of the most enriched gRNAs subsequently tested for 3-HP production. Biosynthesis of 3-HP from malonyl-CoA was obtained by expressing the malonyl-CoA reductase Mcr from Chloroflexus aurantiacus. Shown is the effect of some of the most significant gRNAs on 3-HP titer, i.e., those increasing by more than 15\% the total 3-HP levels normalized to OD and control (no gRNA expressed). Values were obtained in triplicates grown in defined minimal medium with $20 \mathrm{~g} / \mathrm{L}$ glucose, and cultures were sampled after $72 \mathrm{~h}$. Displayed are averages $\pm \mathrm{SD} * * p$ value $<0.01$; $p$ value $<0.05$ (Student's $t$ test: one-tailed, two-sample equal variance). (d) Influence of different gRNAs on the activity of the respective target promoter. Shown are TKL2 \#7 (TSS -147); and ALD2 \#1 (TSS -241), \#7 (TSS -241), \#9 (TSS -251), and \#12 (TSS -192). Since ALD2 \#1 (TSS -241) and \#7 (TSS -241) target the exact same position, a plus and minus sign indicate which strand they bind. (e) Metabolic map of some of the targeted genes and their respective pathways.

can be coupled to transcription factor domains, e.g., the tripartite activator VPR, for efficiently upregulating genes. ${ }^{7,12,17}$ Previous reports have also established the ability to achieve downregulation with dCas9-VPR when targeting close to the TSS. ${ }^{13}$ While graded transcriptional patterns can be achieved based on where the dCas9 complex binds in the promoter region, correct prediction of gene regulation remains challenging as several parameters can potentially affect the expected interference, e.g., the distance to the transcription start site (TSS), condition-dependent presence of other transcription factors, or chromatin accessibility. ${ }^{7,18}$ Thus, in order to both counteract the uncertainties that influence gRNA regulatory efficiency and reach optimal transcriptional interference, we sought to design up to 21 gRNAs per target gene, including up to five adjacent to their respective TSS, yielding a library of 3194 gRNAs $^{18,19}$ (Table S2). The subsequent library was cloned under an anhydrotetracycline (aTc) inducible RNA polymerase III promoter into a centromeric plasmid encoding dCas9 coupled to a VPR activator domain through homologous recombination in vivo after transformation of an S. cerevisiae CEN.PK 11C strain expressing a deregulated Accl in the ethanol phase (Figure S1). ${ }^{7}$ Acc1 is inhibited by Snf1 phosphorylation, and the expression of a double mutant Acc1 $1^{\mathrm{S659A} / \mathrm{S1} 157 \mathrm{~A}}$ with two removed phosphorylation sites, here shortened $\mathrm{Accl}^{* *}$, has been shown to increase production of malonyl-CoA and fatty acid-derived products. ${ }^{20,21}$ This strain background was chosen in order to channel potential increases of the acetyl-CoA pool toward the malonyl-CoA pool and thereby triggering the malonyl-CoA biosensor response. For constructing the biosensor, the malonyl-CoA responsive transcription factor FapR from Bacillus subtilis was employed. FapR binding sites ( fapO) were integrated into a TEF1 promoter (TEF1p) to control GFP expression as the output signal. ${ }^{10}$ FapR will bind to fapO without malonyl-CoA and detach when bound to malonyl-CoA. Thus, the more malonyl-CoA is accumulated the more GFP is expressed. We combined our gRNA library with this malonyl-CoA biosensor, which allows screening for gRNAs and respective regulated genes that potentially contribute to enhancing the flux toward cytosolic acetyl-CoA and its direct derivative, malonyl-CoA. The yeast library was consecutively sorted, regrown and enriched over 3 days using two different fluorescence gates (Figure S1). Next-generation sequencing (NGS) was performed at each stage and the gRNA distribution was determined (Figure 2a,b, Figure S2, Table S5). We subsequently selected 49 gRNAs that were significantly enriched throughout the different sorting steps, targeting a total of 46 genes (Figure S2, Table S4-S5).

Fine-Tuning with CRISPR Technology Reveals New Gene Targets for Metabolic Engineering. From the identified gene targets, we selected 14 genes and coupled their respective promoters (1000 bp upstream of the start codon) to GFP expression, and used the fluorescence levels as a proxy for measuring the degree of gRNA transcriptional interference (Figure 2e, Table S5). Here, expectedly most of the gRNAs showed an upregulating effect when expressed, e.g., 
ALD2 gRNA \#1 (TSS -241) and \#12 (TSS -192), and ALD6 \#13 (TSS -267) (Figure S3). The enriched gRNAs targeting PDX1 (TSS -504) and TKL2 (TSS -147) led to a decrease in GFP expression, in accordance with our in silico predictions (Table S5). By contrast, several gRNAs displayed an opposite regulatory effect to the model prediction, e.g., upregulation for RNR4 \#12 (TSS -497) and downregulation for SLC1 \#20 (TSS -559). Notably, except slightly for ALD6 \#13 (TSS -267), expression of the gRNAs did not lead to any growth impairment (Figure S4).

Next, we reverse engineered the parental strain using the most enriched gRNAs and validated their efficacy by coupling their expression to the production of $3-\mathrm{HP}$, which is directly derived from malonyl-CoA in a two-step reaction catalyzed by the bifunctional malonyl-CoA reductase Mcr from Chloroflexus aurantiacus. ${ }^{20}$ By expressing the Mcr enzyme, we established a direct metabolic pull that can convert any excess malonylCoA/acetyl-CoA into 3-HP accumulation over time. While significantly enriched throughout the screening, many gRNAs did not ultimately increase the overall 3-HP yields, e.g., CRC1 \#6 (TSS -203), ALD6 \#13 (TSS -267), or BAP2 \#10 (TSS -574) (Table S4). Notably, mutations in the FapR operator $(\mathrm{fapO})$ sites within the biosensor construct were not seen for individual clones at the end of the screening. Thus, one can speculate that these gRNAs can still potentially increase (temporally) the malonyl-CoA/acetyl-CoA levels but not sufficiently to be converted to 3-HP (Figure S3). Interestingly, efficient gRNAs, i.e., those ultimately increasing 3-HP levels, target genes whose corresponding enzymes utilize $\mathrm{NAD}^{+}$as the main cofactor, e.g., cytoplasmic aldehyde dehydrogenase Ald2, glyceraldehyde-3-phosphate dehydrogenase Tdh2, or increase the flux toward acetyl-CoA, e.g., Acs2, Ald2, Pdx1 (Figure 2c, Table S4). Unexpectedly, while ALD6 has previously been reported as an efficient target for overexpression to increase 3HP yields, targeting this gene (gRNA TSS -267) significantly lowered the final 3-HP yields (Figure S3-S4, Table S4). ${ }^{22}$ On the other hand, targeting ALD2 gRNA \#1 (TSS -241) ultimately increased $3-\mathrm{HP}$ yield by $27 \%$ compared to the control (Figure 2d, Table S4). ALD2 is repressed in the glucose phase, ${ }^{23}$ and we speculate that in a context where $\mathrm{NAD}^{+}$dependent enzymes seem to be favored for 3-HP production, derepressing it and making it the main aldehyde dehydrogenase, i.e., partially replacing $\mathrm{NADP}^{+}$-dependent ALD6, together allowed a significant effect on 3-HP production. The largest increase in 3-HP production (36\%) was achieved by targeting the gene encoding adenylate kinase 1, ADK1 \#15 (TSS -761), which reversibly converts two ADPs to AMP and ATP. This gRNA increased 3-HP yields by $36 \%$ compared to the control strain (Figure 2) (Table S4), ${ }^{24}$ and led to a downregulating effect on the activity of the $A D K 1$ promoter coupled to GFP (Figure S3). Upon closer inspection, we noticed that the gRNA also binds downstream of the HTA1 coding sequence. HTA1 encodes one of the main histone proteins involved in the structure of chromatin in eukaryotic cells. Notably, ARO4 \#18 (TSS -641) expression led to a $22 \%$ increase in 3-HP yields but did not influence ARO4 transcriptional levels (Table S4, Figure S3). Here, we noticed that ARO4 \#18 (TSS -641) binds close to an adjacent gene of unknown function, SPO23 (YBR250W), and its effect on the SPO23 promoter coupled to GFP was a significant upregulation (Figure S3). Our study also identified new candidate genes to increase 3-HP production such as genes involved in the processing of precursors upstream of malonyl-
CoA, e.g., TKL2 encoding transketolase, and PDX1 encoding a subunit of the pyruvate dehydrogenase complex as well as AHP1 encoding a peroxiredoxin that protects against oxidative stress (Figure 2d). Notably, gRNAs targeting the same promoter at different position also led to different outcomes in 3-HP production, e.g., ALD2 gRNA \#1 (TSS -241) and \#12 (TSS -192) leading to a $27 \%$ and $20 \%$ improvement, or AHP1 gRNA\#6 (TSS -81) and \#10 (TSS -86) being responsible for a $21 \%$ and $14 \%$ increase, respectively (Table S4).

We sought to validate the benefit of utilizing CRISPR for fine-tuning and compared three of the selected upregulating gRNAs against expressing the targeted genes under the strong constitutive TEF1 promoter (TEF1p) (Figure S5). Here, expressing $A L D 2$ under TEF1p did not lead to a significant improvement in 3-HP yield compared to the results with the respective gRNA, further indicating a potential benefit of finetuning with the utilization of dCas9-based transcriptional regulation over classical approaches. As with ALD6 \#13 (TSS -267), TEF1p-ALD6 significantly decreased the overall 3-HP yields. Expectedly, ADK1 expressed under TEF1p did not show any effect on 3-HP yields, which corroborates that a regulation based on ADK1 \#15 (TSS -761) is occurring elsewhere.

\section{DISCUSSION}

While CRISPR technology has been used for industrial applications, ${ }^{25}$ our study reports the first utilization of combining a transcription factor-based biosensor with the versatility and fine-tuning properties of CRISPR-based transcriptional regulation in yeast. We assessed the impact of finetuning gene expression through a gRNA library targeting promoters of computationally predicted candidates for malonyl-CoA production. For this, we used FBA to predict ideal levels for enzymes of a particular pathway based on various parameters, e.g., growth on glucose/ethanol, for a desirable production of cytosolic malonyl-CoA. These analyses ultimately scored genes that are expected to affect fluxes toward the production of malonyl-CoA at different growth rates, and subsequently reduced the number of genes to target. It allowed us to specifically target a defined subset of genes for expression fine-tuning via dCas9-based regulation framework. Most of the predicted genes were not represented among the enriched gRNAs, which highlights a need for improving (i) the score associated with these genes, i.e., most of the highly relevant genes were not in the top 10 score, (ii) the relevance of the suggested genes, i.e., many of them did not have any gRNA enriched, and (iii) a more robust dCas9-based system to achieve correct downregulation or upregulation. Future simulations could benefit from results experimentally obtained to further refine simulation scores. Particularly in the context of expression fine-tuning, it remains challenging to predict this both computationally and experimentally. Additionally, by designing up to 21 gRNAs per promoter we sought to cover potential functional sites in the promoter, that could severely affect transcription levels of the targeted gene. Mapping the regulatory effect of each gRNA targeted to a transcription factor binding sites could eventually provide more insight into the importance of each transcription factor in the targeted promoter. Indeed, several promoter regions are yet to be fully characterized in terms of length and transcription factor binding sites. Interestingly, ALD2 gRNAs \#7 and \#1 show different transcriptional regulation while both being located at TSS -241 but in the opposite direction. While most of the 
gRNAs leading to an upregulating pattern in the vicinity of -200 to $-300 \mathrm{bp}$ from the TSS in accordance with previous reports, ${ }^{7,26}$ the downregulating pattern with dCas9-VPR remains less obvious (Figure S6, Table S7). In addition, employing gRNAs that extensively cover the targeted promoters revealed nonobvious transcriptional regulation that ultimately significantly increased 3-HP production, e.g., SPO23 and HTA1. For HTA1, we speculate that the binding might have reduced HTA1 mRNA levels by blocking RNA polymerase II and thus potentially changed chromatin structures in the genome.

Harnessing the effectiveness of the malonyl-CoA biosensor allows, through multiple rounds of FACS, to retrieve gRNAs that significantly increase fluxes toward malonyl-CoA production. Importantly, FACS screening only captures what happens at the particular moment of sampling, which removes potential bias toward genes favoring growth in general. However, this approach also enriches for gRNAs that are only beneficial at the particular moment in the cultivation and might not show overall benefits e.g., in 3-HP production.

In conclusion, combining a targeted gRNA library and a malonyl-CoA responsive intracellular biosensor in yeast enabled optimal gene expression of nonobvious genes contributing to enhancing the flux toward cytosolic malonylCoA. We envision that our framework for model-assisted finetuning through dCas9-based regulation framework will pave the way for other metabolic engineering studies optimizing the microbial production of a broad range of industrially relevant compounds. Thus, to facilitate this process for the metabolic engineering community, we built an R Shiny app which, as with our study, allows users to select an endogenous metabolite of choice to optimize its production. On the basis of FBA used within this study it then generates both a list of genes to transcriptionally regulate as well as a gRNA library targeting the promoter regions of the suggested genes (publicly hosted by the shinyapps.io server at https://raphdl.shinyapps. io/crispri-gem/).

\section{MATERIAL AND METHODS}

Plasmid Constructions. pFDA09 (TEF1p-BS123-GFP w/ NLS-fapR) ${ }^{10}$ constructed by David et al. was used as the malonyl-CoA biosensor plasmid and the positive control of the malonyl-CoA biosensor, respectively (Table S1). The plasmid pDTU-113 was used for all the dCas9-based experiments. ${ }^{7}$ pDTU-113 contains a gene encoding dCas9 fused with VPR activator, a TetR repressor gene and a gRNA cloning site under the control of the RNA pol III promoter pRPR1 with a tetO operator. The TetR-tet $O$ system enables inducible gRNA expression, and gRNAs transcription was induced by the addition of anhydrotetracycline ( $\mathrm{aTc}$ ) to the growth medium. The gRNA library oligos were ordered from Twist Bioscience (San Francisco, CA, USA) and were amplified with the primers gRNA-F and gRNA-R (Table S1). The PCR amplified library was cloned directly in yeast cells following the high-efficiency transformation protocol developed by Benatuil and colleagues (Figure S1a). ${ }^{27}$ Approximately $4 \mu \mathrm{g}$ of digested backbone and $12 \mu \mathrm{g}$ of PCR amplified gRNA library were used for transformation of a $100 \mathrm{~mL}$ fresh yeast culture of $\mathrm{OD}_{600} \sim$ 1.6. The library was grown on appropriate selective plates and the aim was to take a number of colonies at least three times larger than the library size, to ensure full coverage.

Strain Constructions. CEN.PK113-11C (MATa SUC2$M A L 2-8 c$ his $3 \Delta 1$ ura3-52; P. Kötter, University of Frankfurt
Germany) $)^{28}$ was utilized as the background strain for all the experiments (Table S1). HXT7p-ACC $1 * *$ strain was obtained from Gossing and colleagues. ${ }^{29}$ For constructing MCR01 the malonyl-CoA reductase, $m c r$ gene, was received by $\mathrm{Li}$ et al. (unpublished data). The expression of the bifunctional MCR has been separated into two subdomains. The C-terminal MCR was expressed downstream of a HXT7 promoter and Nterminal MCR under a TDH3 promoter with an UAS upstream of the promoter. The two subdomains were amplified with the primers MCR_part1 (fw/rv) and MCR_part2 (fw/rv) (Table S1). The amplified MCR was genetic integrated into XI-3 in the S. cerevisiae genome, with CRISPR/Cas9 technique.

DELFT medium was used as a minimal synthetic medium for yeast growth. DELFT composition was $7.5 \mathrm{~g} / \mathrm{L}$ $\left(\mathrm{NH}_{4}\right)_{2} \mathrm{SO}_{4}, 14.4 \mathrm{~g} / \mathrm{L} \mathrm{KH}_{2} \mathrm{PO}_{4}, 0.5 \mathrm{~g} / \mathrm{L} \mathrm{MgSO}_{4} \cdot 7 \mathrm{H}_{2} 0,22 \mathrm{~g} /$ $\mathrm{L}$ dextrose, $2 \mathrm{~mL} / \mathrm{L}$ trace metals solution, and $1 \mathrm{~mL} / \mathrm{L}$ vitamins. ${ }^{30}$ The $\mathrm{pH}$ was adjusted to 6 . Liquid cultures of both microorganisms were grown aerobically at $30{ }^{\circ} \mathrm{C}$ in shake flasks with a shaking speed of $200 \mathrm{rpm}$.

Flux Balance Analysis. Flux balance analysis (FBA) was performed using the consensus genome-scale metabolic model of yeast as it was revised by Aung et al. 2013 (Yeast v7.6). ${ }^{14,31}$ The analysis was performed for two carbon sources: glucose and ethanol. Once the maximum specific growth rate $\left(\mu_{\max }\right)$ for each carbon source was identified, 11 suboptimal growth rates were applied to the model, in the interval $0.3 \times \mu_{\max }$ to $0.8 \times$ $\mu_{\max }$ in increments of $0.05 \times \mu_{\max }$. For each suboptimal growth rate, two analyses were performed, one for maximum cytosolic acetyl production and one for maximum cytosolic malonyl production. In all simulations parsimonious FBA (pFBA) was performed, meaning that the solution for each analysis was the one with the minimal sum of fluxes. ${ }^{32}$

For each reaction and condition, a $k$-score was calculated as $k=v / v_{\mu \max }$ in a fashion similar to previous studies. ${ }^{33}$ This score compares the flux of each reaction in each simulation with the flux of the same reaction under maximum growth rate conditions. Therefore, reactions that show $k$-scores $>1$ have an increase in the corresponding flux compared to the $\mu_{\max }$ condition and $k$-scores $<1$ have a decrease in the corresponding flux. The maximum $k$-score was arbitrarily set to 100 for $k$ scores that are infinite (i.e., when $v_{\mu \max }=0$ ). Reactions that showed inconsistencies between the 11 different suboptimal growth rates (i.e., some $k<1$ and some $k>1$ ) were filtered out. Afterward, reactions were associated with the genes that encode the enzyme(s) that catalyze each reaction. In terms of gene expression, it was assumed that a $k$-score $>1$ represented upregulation and a $k$-score $<1$ represented downregulation of the gene expression levels. For genes that take part in more than one reaction, average $k$-scores were calculated. Genes that showed average $k$-scores between 0.549 and 1.001 were ruled out as nonsignificantly changed. The value of 0.549 was chosen because it corresponds to the value of a flux that decreases proportionally to the decrease in biomass production $((0.3+$ $0.35+\ldots+0.8) / 11)$ and not to any additional increase toward acetyl/malonyl. Finally, FVA was computed for all cases to show if any genes were associated with reactions that exhibited flux variability, which leads to $k$-scores that vary depending on the flux variability and could correspond to false positives. ${ }^{15}$ Said genes were marked as such in Table S2.

Library Design. For each gene retrieved from the FBA, 15-21 gRNAs were designed using CRISPR-ERA and Yeast CRISPRi. ${ }^{18,19}$ The binding site of each gRNA was identified by the distance from the transcription start site (TSS). ${ }^{19}$ For each 
gene, $2-5$ gRNAs were designed to target a window from -50 to +50 from the TSS. The total library contained 3194 gRNAs in total.

Fluorescence Measurements and Sorting. For realtime monitoring of GFP expression, a BioLector was used (m2p-laboratories $\mathrm{GmbH}$, Baesweiler, Germany). The cultures were grown in using FlowerPlates, the total volume of each culture was $1 \mathrm{~mL}$, and initial $\mathrm{OD}_{600}$ was $0.05-0.1$. GFP expression levels were measured by the ratio of green fluorescence/biomass. For the FACS assays, the FACS Aria cell sorter was used (BD Biosciences, San Jose, CA, USA). Cryostocks of the yeast library and control (no library) were grown in synthetic minimal media at an initial $\mathrm{OD}_{600}=0.1$. After $3 \mathrm{~h}$ of culture, aTc $(1000 \times)$ was added to induce gRNA expression. At $7 \mathrm{~h}$, a sample from the culture was taken for $\mathrm{OD}_{600}$ measurement and FACS. An initial round of 20000 events was performed to evaluate the overall fluorescence difference between the library and the control (Figure S1b). Two gates were subsequently defined for sorting, namely P2 that contained cells that showed medium to high fluorescence (approximately top 2\% of the population) and P3 that contained cells with extremely high fluorescence (approximately top $0.2 \%$ of the population) (Figure S1b).

Next-Generation Sequencing Analysis. After each round of sorting, plasmids of the sorted yeast libraries were extracted using Zymoprep Yeast Plasmid Miniprep (Zymo Research, CA, USA). Libraries were prepared based on Illumina DNA Nextera Sequencing and next-generation sequencing was performed on a MiSeq Benchtop Sequencer (Illumina, San Diego, CA) as described in Lee et al. ${ }^{34}$ Fastq sequences were analyzed using a custom $\mathrm{R}$ script. The sequences between the end of RPR1p (5'-CGATTGGCAG) and beginning of RPR1t (5'-GTTTTAGAGC) were probed and matched to the gRNA library. The number of hits, i.e., the number of times a gRNA is matched to the gRNA library, was quantified and normalized by the total number of NGS read counts for each run. The most enriched gRNAs were retrieved by taking the base $2 \log$ of the ratio of the normalized gRNA hits of each sorting over the NGS from the preculture, ${ }^{35}$ and plotted using the R library ggplot2 3.3.0.

3-Hydroxypropionic Acid Measurements. All culture samples were centrifuged at $12000 \mathrm{~g}$, and the supernatants were diluted 1:5 with $0.5 \mathrm{mM} \mathrm{H}_{2} \mathrm{SO}_{4}$. The concentration of 3$\mathrm{HP}$ was conducted with high-performance liquid chromatography (HPLC, Dionex UltiMate 3000; Thermo Fisher Scientific, Waltham, MA, USA), equipped with an Aminex HPX-87H (Bio-Rad, Hercules, USA) column at $65^{\circ} \mathrm{C}$. Each sample was analyzed with a mobile phase of $0.5 \mathrm{mM} \mathrm{H}_{2} \mathrm{SO}_{4}$ at a flow rate of $0.5 \mathrm{~mL} / \mathrm{min}$ for $35 \mathrm{~min}$.

R Shiny App. An app was built using the R programming language and the $\mathrm{R}$ Shiny application framework (http:// CRAN.R-project.org/package=shiny). The complete list of gRNAs was previously obtained from Yeast CRISPRi and integrated into the app. ${ }^{18}$

\section{ASSOCIATED CONTENT}

\section{S Supporting Information}

The Supporting Information is available free of charge on the ACS Publications website at DOI: 10.1021/acssynbio.9b00258.

Supplementary Tables: S. cerevisiae strains, plasmids, and oligos used in this study (Table S1); gRNA library and in silico rate info obtained from the FBA (Table S2); GO Term enrichment of the 168 computationally retrieved genes (Table S3); 3-HP quantification (Table S4); Fluorescence levels measuring the degree of gRNA transcriptional interference (Table S5); gRNA distribution for each FACS sorting (Table S6); Summary of the TSS distance, strand, and transcriptional regulation for the promising gRNAs (Table S7) (XLSX)

Methods and Materials and Supplementary Figures S1S6 (PDF)

\section{AUTHOR INFORMATION}

\section{Corresponding Author}

*E-mail: davidfl@chalmers.se.

ORCID $\odot$

Raphael Ferreira: 0000-0001-9881-6232

\section{Author Contributions}

RF and FD conceived the study. RF, CS, and AH carried out the experiments. BS performed the in silico simulations. RF analyzed the data and wrote the manuscript. RF and BS built the R Shiny app. JN, VS, and FD supervised the study. JN and FD acquired funding. All authors contributed to the analysis and the discussion of the results. All authors read and approved the final manuscript.

Notes

The authors declare no competing financial interest.

\section{ACKNOWLEDGMENTS}

The authors would like to thank Xiaowei Li for providing the mor expression system, Ahmed Ali for valuable scientific discussions, as well as Paulo Teixeira and Tyler Doughty for reviewing the manuscript. This work was funded by the Novo Nordisk Foundation (grant no. NNF10CC1016517), Swedish Foundation for Strategic Research, ÅForsk (Ångpanneföreningens forskningsstiftelse) research, European Union's Horizon 2020 (grant number 686070), FORMAS, and the Knut and Alice Wallenberg Foundation.

\section{REFERENCES}

(1) Nielsen, J., and Keasling, J. D. (2016) Engineering Cellular Metabolism. Cell 164 (6), 1185-1197.

(2) Marella, E. R., Holkenbrink, C., Siewers, V., and Borodina, I. (2018) Engineering Microbial Fatty Acid Metabolism for Biofuels and Biochemicals. Curr. Opin. Biotechnol. 50, 39-46.

(3) Williams, T. C., Pretorius, I. S., and Paulsen, I. T. (2016) Synthetic Evolution of Metabolic Productivity Using Biosensors. Trends Biotechnol. 34 (5), 371-381.

(4) Kim, I.-K., Roldão, A., Siewers, V., and Nielsen, J. (2012) A Systems-Level Approach for Metabolic Engineering of Yeast Cell Factories. FEMS Yeast Res. 12 (2), 228-248.

(5) Teixeira, P. G., Ferreira, R., Zhou, Y. J., Siewers, V., and Nielsen, J. (2017) Dynamic Regulation of Fatty Acid Pools for Improved Production of Fatty Alcohols in Saccharomyces Cerevisiae. Microb. Cell Fact. 16 (1), 45.

(6) Zhang, C., and Hua, Q. (2016) Applications of Genome-Scale Metabolic Models in Biotechnology and Systems Medicine. Front. Physiol., DOI: 10.3389/fphys.2015.00413.

(7) Jensen, E. D., Ferreira, R., Jakočiūas, T., Arsovska, D., Zhang, J., Ding, L., Smith, J. D., David, F., Nielsen, J., Jensen, M. K., et al. (2017) Transcriptional Reprogramming in Yeast Using DCas9 and Combinatorial GRNA Strategies. Microb. Cell Fact. 16 (1), 46.

(8) Farzadfard, F., Perli, S. D., and Lu, T. K. (2013) Tunable and Multifunctional Eukaryotic Transcription Factors Based on CRISPR/ Cas. ACS Synth. Biol. 2 (10), 604-613. 
(9) Zha, W., Rubin-Pitel, S. B., Shao, Z., and Zhao, H. (2009) Improving Cellular Malonyl-CoA Level in Escherichia Coli via Metabolic Engineering. Metab. Eng. 11 (3), 192-198.

(10) David, F., Nielsen, J., and Siewers, V. (2016) Flux Control at the Malonyl-CoA Node through Hierarchical Dynamic Pathway Regulation in Saccharomyces Cerevisiae. ACS Synth. Biol. 5 (3), 224233.

(11) Johnson, A. O., Gonzalez-Villanueva, M., Wong, L., Steinbüchel, A., Tee, K. L., Xu, P., and Wong, T. S. (2017) Design and Application of Genetically-Encoded Malonyl-CoA Biosensors for Metabolic Engineering of Microbial Cell Factories. Metab. Eng. 44, 253-264.

(12) Chavez, A., Scheiman, J., Vora, S., Pruitt, B. W., Tuttle, M., Iyer, E. P. R., Lin, S., Kiani, S., Guzman, C. D., Wiegand, D. J., et al. (2015) Highly Efficient Cas9-Mediated Transcriptional Programming. Nat. Methods 12 (4), 326-328.

(13) Kiani, S., Chavez, A., Tuttle, M., Hall, R. N., Chari, R., TerOvanesyan, D., Qian, J., Pruitt, B. W., Beal, J., Vora, S., et al. (2015) Cas9 GRNA Engineering for Genome Editing, Activation and Repression. Nat. Methods 12 (11), 1051-1054.

(14) Aung, H. W., Henry, S. A., and Walker, L. P. (2013) Revising the Representation of Fatty Acid, Glycerolipid, and Glycerophospholipid Metabolism in the Consensus Model of Yeast Metabolism. Ind. Biotechnol. 9 (4), 215-228.

(15) Mahadevan, R., and Schilling, C. H. (2003) The Effects of Alternate Optimal Solutions in Constraint-Based Genome-Scale Metabolic Models. Metab. Eng. 5 (4), 264-276.

(16) Hong, E. L., Balakrishnan, R., Dong, Q., Christie, K. R., Park, J., Binkley, G., Costanzo, M. C., Dwight, S. S., Engel, S. R., Fisk, D. G., et al. (2007) Gene Ontology Annotations at SGD: New Data Sources and Annotation Methods. Nucleic Acids Res. 36, D577-D581.

(17) Qi, L. S., Larson, M. H., Gilbert, L. A., Doudna, J. A., Weissman, J. S., Arkin, A. P., and Lim, W. A. (2013) Repurposing CRISPR as an RNA-Guided Platform for Sequence-Specific Control of Gene Expression. Cell 152 (5), 1173-1183.

(18) Smith, J. D., Suresh, S., Schlecht, U., Wu, M., Wagih, O., Peltz, G., Davis, R. W., Steinmetz, L. M., Parts, L., and St. Onge, R. P. (2016) Quantitative CRISPR Interference Screens in Yeast Identify Chemical-Genetic Interactions and New Rules for Guide RNA Design. Genome Biol. 17 (1), 45.

(19) Liu, H., Wei, Z., Dominguez, A., Li, Y., Wang, X., and Qi, L. S. (2015) CRISPR-ERA: A Comprehensive Design Tool for CRISPRMediated Gene Editing, Repression and Activation. Bioinformatics 31 (22), 3676-3678.

(20) Shi, S., Chen, Y., Siewers, V., and Nielsen, J. (2014) Improving Production of Malonyl Coenzyme A-Derived Metabolites by Abolishing Snf1-Dependent Regulation of Acc1. mBio, DOI: $10.1128 / \mathrm{mBio} .01130-14$.

(21) Ferreira, R., Teixeira, P. G., Gossing, M., David, F., Siewers, V., and Nielsen, J. (2018) Metabolic Engineering of Saccharomyces Cerevisiae for Overproduction of Triacylglycerols. Metab. Eng. Commun. 6, 22-27.

(22) Chen, Y., Bao, J., Kim, I.-K., Siewers, V., and Nielsen, J. (2014) Coupled Incremental Precursor and Co-Factor Supply Improves 3Hydroxypropionic Acid Production in Saccharomyces Cerevisiae. Metab. Eng. 22, 104-109.

(23) Aranda, A., and del Olmo, M. 1. (2003) Response to Acetaldehyde Stress in the YeastSaccharomyces Cerevisiae Involves a Strain-Dependent Regulation of SeveralALD Genes and Is Mediatedby the General Stress Response Pathway. Yeast 20 (8), $747-759$.

(24) Jayachandran, C., Athiyaman, B. P., and Sankaranarayanan, M. (2017) Cofactor Engineering Improved CALB Production in Pichia Pastoris through Heterologous Expression of NADH Oxidase and Adenylate Kinase. PLoS One 12 (7), No. e0181370.

(25) Ferreira, R., David, F., and Nielsen, J. (2018) Advancing Biotechnology with CRISPR/Cas9: Recent Applications and Patent Landscape. J. Ind. Microbiol. Biotechnol. 45 (7), 467-480.
(26) Jensen, M. K. (2018) Design Principles for Nuclease-Deficient CRISPR-Based Transcriptional Regulators. FEMS Yeast Res., DOI: $10.1093 /$ femsyr/foy039.

(27) Benatuil, L., Perez, J. M., Belk, J., and Hsieh, C.-M. (2010) An Improved Yeast Transformation Method for the Generation of Very Large Human Antibody Libraries. Protein Eng., Des. Sel. 23 (4), 155159.

(28) van Dijken, J. P., Bauer, J., Brambilla, L., Duboc, P., Francois, J. M., Gancedo, C., Giuseppin, M. L. F., Heijnen, J. J., Hoare, M., Lange, H. C., et al. (2000) An Interlaboratory Comparison of Physiological and Genetic Properties of Four Saccharomyces Cerevisiae Strains. Enzyme Microb. Technol. 26 (9), 706-714.

(29) Gossing, M., Smialowska, A., and Nielsen, J. (2018) Impact of Forced Fatty Acid Synthesis on Metabolism and Physiology of Saccharomyces Cerevisiae. FEMS Yeast Res., DOI: 10.1093/femsyr/ foy096.

(30) Verduyn, C., Postma, E., Scheffers, W. A., and Van Dijken, J. P. (1992) Effect of Benzoic Acid on Metabolic Fluxes in Yeasts: A Continuous-Culture Study on the Regulation of Respiration and Alcoholic Fermentation. Yeast 8 (7), 501-517.

(31) Orth, J. D., Thiele, I., and Palsson, B. Ø. (2010) What Is Flux Balance Analysis? Nat. Biotechnol. 28 (3), 245-248.

(32) Lewis, N. E., Hixson, K. K., Conrad, T. M., Lerman, J. A., Charusanti, P., Polpitiya, A. D., Adkins, J. N., Schramm, G., Purvine, S. O., Lopez-Ferrer, D., et al. (2010) Omic Data from Evolved E. Coli Are Consistent with Computed Optimal Growth from Genome-Scale Models. Mol. Syst. Biol. 6 (1), 390.

(33) Choi, H. S., Lee, S. Y., Kim, T. Y., and Woo, H. M. (2010) In Silico Identification of Gene Amplification Targets for Improvement of Lycopene Production. Appl. Environ. Microbiol. 76 (10), 30973105 .

(34) Lee, J. S., Kallehauge, T. B., Pedersen, L. E., and Kildegaard, H. F. (2015) Site-Specific Integration in CHO Cells Mediated by CRISPR/Cas9 and Homology-Directed DNA Repair Pathway. Sci. Rep. 5, 8572.

(35) Joung, J., Konermann, S., Gootenberg, J. S., Abudayyeh, O. O., Platt, R. J., Brigham, M. D., Sanjana, N. E., and Zhang, F. (2017) Genome-Scale CRISPR-Cas9 Knockout and Transcriptional Activation Screening. Nat. Protoc. 12 (4), 828-863. 